\title{
Effective Inhibition of Xenografts of Hepatocellular Carcinoma (HepG2) by Rapamycin and Bevacizumab in an Intrahepatic Model
}

\author{
Lai-Chun Ong, ${ }^{1}$ In-Chin Song, ${ }^{1}$ Yi Jin, ${ }^{1}$ Irene H. C. Kee, ${ }^{1}$ Eeyan Siew, ${ }^{1}$ Sidney Yu, ${ }^{1}$ \\ Choon-Hua Thng, ${ }^{2}$ Hung Huynh, ${ }^{2}$ Pierce K. H. Chow ${ }^{1,2,3,4}$ \\ ${ }^{1}$ Singapore General Hospital, Outram Road, Singapore 169608, Singapore \\ ${ }^{2}$ National Cancer Centre, 11 Hospital Drive, Singapore 169610, Singapore \\ ${ }^{3}$ Duke-NUS Graduate Medical School, Singapore, Singapore \\ ${ }^{4}$ Department of General Surgery, Singapore General Hospital, Singapore 169608, Singapore
}

\begin{abstract}
Purpose: Hepatocellular carcinoma (HCC) displays a characteristic hypervascularity and depends on angiogenesis for tumor growth, which thus provides a potential target for therapeutic approaches to HCC. In this study, through the use of combined micro-positron emission tomography (PET)/computed tomography (CT), we investigate if such a combined targeting of vascular endothelial growth factor (VEGF) activity and expression might retard HCC growth in an orthotopic intrahepatic xenograft model.

Procedures: Xenograft models were created by intraportal vein injection of HepG2 cell suspensions in severe combined immunodeficient mice. The mice were then treated with (1) rapamycin (RAPA), a mammalian target of rapamycin pathway inhibitor; (2) bevazicumab (BEV), a VEGF monoclonal antibody; and (3) a RAPA/BEV combination.

Results: Assessment of HCC progression using CT with Omnipaque and PET with 2-deoxy-2(F-18)-fluoro-D-glucose showed that mice treated with RAPA/BEV had the lowest standardized uptake values (SUVs). At week 2, mice treated with RAPA/BEV, RAPA, and BEV all showed a marked decrease in the SUV ${ }_{\text {max }}$ readings with the greatest drop being observed in the RAPA/ BEV group $(1.33+0.26,1.81+0.2,2.05+0.4$ vs. vehicle control $2.11+0.53)$.

Conclusions: Our results, supported by micro-PET/CT, suggest that RAPA/BEV represents a potential novel antiangiogenic therapy for the treatment of HCC.
\end{abstract}

Key words: Hepatocellular carcinoma, Orthotopic xenograft model, Tumor vasculature, Rapamycin, Bevacizumab, ${ }^{18}$ F-FDG-PET, Micro-CT, Micro-PET

\section{Introduction}

$\mathrm{H}$ epatocellular carcinoma (HCC) is a hypervascular tumor characterized by neovascularization, which plays an important role in the growth and progression of HCC [1]. It is one of the five most common cancers worldwide, with a particularly high prevalence in Asia due

Correspondence to: Pierce K. H. Chow; e-mail: gsupc@singnet.com.sg to endemic hepatitis B virus infection [2]. Curative treatments such as liver resection and transplantation are only possible if the tumor is detected at an early stage and applies only to a minority of patients [3]. Prognosis after resection, however, remains poor due to high incidence of postoperative recurrence, and in addition, transplantation is often limited by the shortage of grafts [4]. Since conventional cytotoxic chemotherapy has not shown to be effective against $\mathrm{HCC}$ in both the adjuvant and palliative settings, 
the development of a novel effective therapy for HCC will be of significant clinical importance [5].

HCC is one of the most vascular solid tumors and highly dependent on angiogenesis for tumor growth. The potential of antiangiogenic therapy for $\mathrm{HCC}$ is thus particularly enticing. Rapamycin (Sirolimus) is an inhibitor of the kinase mammalian target of rapamycin (mTOR) and possesses both antiproliferative and antiangiogenic properties [6]. The inhibition of mTOR induces cell cycle arrest in G1 phase [7, 8]. Rapamycin also inhibits vascular endothelial growth factor (VEGF) secretion and VEGF signaling in endothelial cells and impairs tumor growth by an antiangiogenic mechanism [9]. Another therapeutic agent with demonstrated anticancer efficacy is the anti-VEGF antibody bevacizumab. Bevacizumab has been studied in metastatic renal and colorectal cancers with promising results [10-12]. However, its effect in HCC is less conclusively documented.

While the use of animal xenograft models is central to pre-clinical proof-of-principle studies in experimental oncology $[13,14]$, the correlation between the results of preclinical studies and those of subsequent clinical trials has been poor [15]. Such in vivo models in oncology research has traditionally been largely restricted to ectopic models which are both easy to create and allows direct access for tumor measurement. The criticism against such ectopic xenograft models is that they may be poorly representative of the milieu under which these tumors exist in the pathophysiological state [16]. While it is possible to create xenograft tumor models in relevant orthotopic locations (such as HCC xenografts in intrahepatic sites), they are both technically challenging to create and there has not been easily practicable ways to perform serial measurement of such tumors [17].

Recent developments in pre-clinical bioimaging have, however, offered a practical means for serial measurement of xenografts in intraperitoneal and other orthotopic locations. Positron emission tomography (PET) imaging with 2-deoxy-2-(F-18)-fluoro-D-glucose $\left({ }^{18} \mathrm{~F}-\mathrm{FDG}\right)$ is widely used in clinical cancer detection because of the increase in metabolism found in many tumors [18, 19], and this has been extended to pre-clinical research [20]. As a lone modality, there are limitations with ${ }^{18} \mathrm{~F}$-FDG PET imaging of HCC with some studies reporting a falsenegative rate approaching $50 \%$ as well as poor anatomical localization [21, 22]. Hence, computed tomography (CT) imaging is an important adjunct to provide confirmatory support in the diagnosis of HCC [23].

To assess whether the combined suppression of both VEGF activity by bevacizumab and VEGF expression by targeting mTOR via rapamycin (RAPA) results in the inhibition of HCC tumor, we created an orthotopic xenograft HCC model. The antitumor effect of RAPA/BEV in xenograft models of HCC was demonstrated by using micro-PET imaging and evaluation of the standardized uptake values (SUV) of the tumors and by micro-CT imaging. Our results provide strong support for the use of RAPA/BEV as novel antiangiogenic therapy for HCC.

\section{Materials and Methods}

\section{Animal Preparation}

All animal experiments were approved by the Institutional Animal Care and Use Committee and carried out in an AAALACaccredited facility. Six- to 8-week-old mice, homozygous for the severe combined immunodeficiency were used. The animals were provided with sterilized food and water ad libitum and housed in negative pressure isolators.

\section{HCC Xenograft Model}

The HCC cell line HepG2 was acquired from the American Type Culture Collection. Cells were cultivated in Eagle's minimum essential medium (MEM) supplemented with 10\% fetal bovine serum and maintained under a $5 \% \mathrm{CO}_{2}$-humidified atmosphere at $37^{\circ} \mathrm{C}$. Cells growing exponentially in vitro were trypsinized and harvested for tumor implantation. All animal manipulations were performed under sterile conditions. To create orthotopic HCC models, mice were anesthetized with intraperitoneal injection of a ketamine/diazepam solution $(50 \mathrm{mg} / \mathrm{kg}$ ketamine and $5 \mathrm{mg} / \mathrm{kg}$ diazepam) and operated on a pre-warmed operation table. A subcutaneous dose of baytril $5 \mathrm{mg} / \mathrm{kg}$ injection was given before operative procedure. Briefly, an upper midline laprotomy was performed. Aided by the use of an operating microscope (Leica, Switzerland), the portal vein was exposed by displacing the duodenum through a midline incision of the abdomen and a suspension of $1 \times 10^{6}$ HepG2 cells in MEM medium was injected via the portal vein using a 30 -gauge needle within a period of a minute. The abdominal incision was sutured close with $5 / 0$ maxon (monofilament polyglyconate synthetic absorbable suture) and skin was closed with $5 / 0$ prolene (polypropylene suture). Postoperatively, the mice were given a subcutaneous injection of $5 \mathrm{mg} / \mathrm{kg}$ caprofen for analgesia. The animals were kept warm and returned to their cages when fully awake. PET scanning was performed on the mice periodically after day 4 .

\section{Micro-PET Imaging Using ${ }^{18} F-F D G$}

Prior to micro-PET imaging, the mice were fasted overnight until imaging was completed, with water being available ad libitum. On the day of imaging, the mice were pre-warmed to a body temperature of $37^{\circ} \mathrm{C}$ before an approximate $150 \mu \mathrm{Ci}$ of ${ }^{18} \mathrm{~F}$-FDG $(0.6 \mathrm{mM})$ was administered intraperitoneally. The body temperature was maintained at $37^{\circ} \mathrm{C}$ throughout the uptake period. Micro-PET imaging was performed with the R4 micro-PET scanner (Concordes Microsystems Inc.) $45 \mathrm{~min}$ after ${ }^{18} \mathrm{~F}-\mathrm{FDG}$ injections. The specification of the R4 micro-PET scanner is briefly as follows: it has a ring diameter of $26 \mathrm{~cm}$ and a 7.8-cm axial field of view and the intrinsic spatial resolution ranges from 1.56 to $2.01 \mathrm{~mm}$, with a mean of $1.75 \mathrm{~mm}$. The reconstructed resolution is $1.8 \mathrm{~mm}$ full width at half maximum in the center of the field of view and $3 \mathrm{~mm}$ at $4 \mathrm{~cm}$ radial offset.

Each mouse was imaged for $10 \mathrm{~min}$. During the micro-PET imaging, the mice were placed in an imaging chamber and kept under isoflurane anesthesia. ${ }^{18}$ F-FDG was obtained from the Department of Nuclear Medicine of Singapore General Hospital. 


\section{Image Reconstruction}

Images were reconstructed using filtered backprojection without scatter or attenuation correction. For cross-calibration of the dose calibrator and the micro-PET scanner, a $25-\mathrm{cm}^{3}$ cylinder phantom filled with known concentration of ${ }^{18}$ F-FDG was imaged. From this scan, a system calibration factor was derived by dividing the known activity concentration in the phantom by the measured mean counts per voxel in the reconstructed PET images.

\section{Quantitative Image Analysis}

Regions of interest were manually drawn over the tumors. Tracer uptake by the various tumors were quantified by maximum standardized uptake values $\left(\mathrm{SUV}_{\max }\right)$ using the formula: $\mathrm{SUV}_{\max }=$ maximum tissue activity concentration $(\mu \mathrm{Ci} / \mathrm{ml}) /$ injected dose $(\mu \mathrm{Ci}) \times$ body weight $(\mathrm{g})$.

\section{Animal Preparation and Micro-CT Imaging}

Images were obtained on a Rigaku micro-CT scanner (JMorita Engineering Co., Ltd, Japan). This is a dedicated CCD plate small animal micro-CT scanner with a bore diameter of $10 \mathrm{~cm}$. The X-ray voltage was set at $90 \mathrm{kVp}$ and an anode current of $88 \mu \mathrm{A}$. Images of the whole animal were obtained after one revolution that lasted $17 \mathrm{~s}$. The minimum pixel size is $48 \times 48 \times 36 \mu \mathrm{m}$. Mice were fasted before the scan to minimize imaging artifacts due to minerals that are found in rodent chow. Three hours prior to the scan, mice were given an intraperitoneal bolus dose of $20 \mathrm{ml} / \mathrm{kg}$ of Omnipaque 300 . The mice received $5 \%$ isoflurane in oxygen during rapid inhalation induction anesthesia (1-2 min). Maintenance of anesthesia during each scan was achieved using $1-2 \%$ isoflurane in oxygen. The total scan time for each scan is 2 min.

\section{Determination of HCC Xenograft Progression in an Orthotopic Intrahepatic Model}

The progression of the orthotopic HCC xenograft models was determined by imaging mice inoculated with HepG2 cells at different time points. Mice were killed at days 0, 4, 7, and 10 after tumor cells inoculation following the completion of imaging on those days. Histology (see below) was performed to correlate tumor progression with micro-PET results. A total of 24 mice were used.

\section{Drug Treatment}

Four days after tumor cell inoculation, HCC xenograft mice were randomized into one of four groups and treated as follows: (1) intraperitoneal injection with $100 \mu \mathrm{l}$ of saline (vehicle/control), (2) oral administration of RAPA (Rapamune, Wyeth Pharmaceuticals Company, Guayama) at $1 \mathrm{mg} / \mathrm{kg}$, (3) intraperitoneal injection of $5 \mathrm{mg} / \mathrm{kg}$ bevacizumab (BEV, Avastin, Genentech, Inc., South San Francisco, CA, USA), and (4) combined oral RAPA and injected BEV (BEV/RAPA). Control and BEV injections were performed on a fortnightly basis while oral RAPA was administered daily. Each group involved 13 independent tumor-bearing mice. Body weights, ascites formation, and overall survival were monitored twice weekly. Tumor-bearing mice were sacrificed when they became moribund and the presence of ascitic fluid was recorded for each mouse. At the end of the treatment regimen, all animals were sacrificed and tumors harvested for histological analysis.

\section{Histology and Immunohistochemical Analysis}

The mice were killed with overdosage of pentobarbitone. Livers were harvested in $4 \%$ buffered formaldehyde for $24 \mathrm{~h}$, processed into paraffin, then sectioned at $3 \mu \mathrm{m}$. Sections were deparaffinized with xylene, degrading alcohol $100 \%, 95 \%$, and $70 \%$, and water, and finally stained with hematoxylin and eosin (H\&E).

For immunoreactivity staining, 5- $\mu \mathrm{m}$ deparaffinized polysincoated sections were treated with $3 \%$ hydrogen peroxide for nonspecific binding. Two types of antibody were employed for immunohistochemical staining. Ki-67, rabbit monoclonal antibody, and vascular endothelial growth factor, epitope-specific rabbit antibodies, were from Lab Vision Corp, USA. The antibody against VEGF could cross-react with both mouse and human proteins. Both were revealed using the peroxidase-antiperoxidase technique by Chemmate Envision Kit HRP/DAB.

\section{Visual Grading of Ki-67 and VEGF Protein Expression}

The expressions of Ki-67 and VEGF proteins within all sections were quantified by a visual grading score [24]. The proliferation index was determined by calculating the number of Ki-67-positive cells per total number of cells in ten fields at $\times 40$ magnification and expressing it as a percentage. All sections were graded on three separate occasions by three different investigators. If the grade varied between scoring runs, the results of the latter analysis were chosen because this was considered more accurate. An overall estimate of the intensity was used rather than quantifying the regions of highest expression.

\section{Statistical Analysis}

Body weight, Ki-67 expression, and SUV differences were analyzed by one-way ANOVA. Differences in survival were evaluated by the Kaplan-Meier method and compared by a log-rank test.

\section{Results}

\section{Progression of HCC Xenograft in an Orthotopic Intrahepatic Model}

Mice inoculated with HepG2 cells via intraportal vein injection were subjected to micro-PET scanning to confirm successful tumor transplantation. Micro-PET scanning showed successful tumor implantation for all inoculated mice. A total of 24 mice were used and randomized into four groups to be sacrificed at various time points. As shown by Fig. 1a, b, by day 4, micro-PET scanning revealed a metabolically active lesion in the liver $\left(\mathrm{SUV}_{\max }=1.88 \pm\right.$ 0.13). By day 7, the SUV $\mathrm{max}_{\max }$ reading had increased to $2.44 \pm$ 0.17 . Subsequent $S_{U V} V_{\max }$ readings continued to rise with the mice becoming increasingly weak clinically. H\&E histological analysis corresponded with the $\mathrm{SUV}_{\max }$ readings thus supporting the use of micro-PET as a diagnostic tool in translational research. 

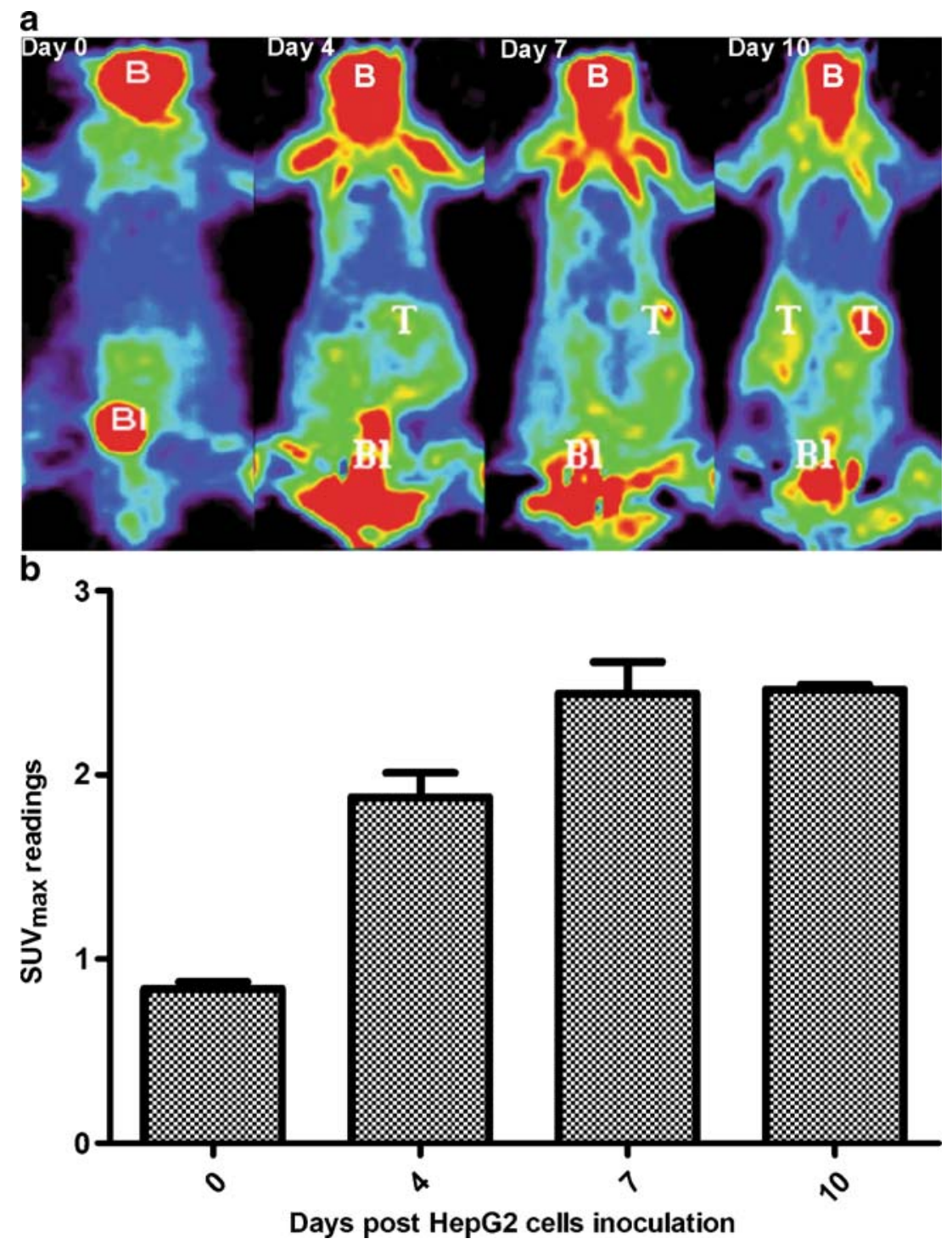

Fig. 1. a Coronal ${ }^{18} \mathrm{~F}-\mathrm{FDG}$ images of representative mice bearing HCC xenograft tumor over time. Images obtained on a dedicated small animal micro-PET scanner depicted tumor progression. At day $0,{ }^{18} \mathrm{~F}$-FDG was mainly taken up by the heart and lungs. However, as tumor progresses, the glucose analogue concentrates in the actively metabolizing HCC. b SUV $\max$ readings of HCC xenograft tumors after inoculation. Error bar shows SEM.

\section{The Use of RAPA/BEV Effectively Inhibits HCC Xenografts as Depicted by Micro-PET}

Based on the results of Fig. 1, we have chosen to commence drug treatment 4 days after tumor cells inoculation. In general, there was a significant reduction ( $p$ value $<0.05$ ) in the $\mathrm{SUV}_{\max }$ readings of the mice undergoing RAPA/BEV treatment when compared to the other three groups of mice (Fig. 2a). One week after tumor cells inoculation, mice treated with RAPA/BEV had the lowest $\mathrm{SUV}_{\max }$ reading of $1.8 \pm 0.29$. Mice treated with RAPA and BEV had a reading of $2.42 \pm$ 0.37 and $2.18 \pm 0.1$, respectively. Vehicle control mice had a $\mathrm{SUV}_{\max }$ reading of $1.9 \pm 0.2$. At week 2, all mice, with the exception of control mice, showed a marked decrease in the $\mathrm{SUV}_{\text {max }}$ readings. Mice treated with
RAPA/BEV had a low $\mathrm{SUV}_{\max }$ reading of $1.33 \pm 0.26$, followed by those treated with RAPA $(1.81 \pm 0.2)$ and BEV (2.05 \pm 0.4$)$. On the other hand, $\mathrm{SUV}_{\max }$ readings of control mice increased from $1.9 \pm 0.2$ to $2.11 \pm 0.5$. From week 3 onwards, mice treated with single drug agent (RAPA or BEV) showed significantly higher $\mathrm{SUV}_{\max }$ readings compared to vehicle control. Such higher readings were, however, contributed by a very small volume of the tumor cells as indicated by micro-PET analysis. We speculate that this could be due to widespread necrosis that was observed in the control mice, thus accounting for the lower $\mathrm{SUV}_{\max }$ readings. Despite this, at week 3 , there was still a $43.0 \pm 5.2 \%$ and at week 4 , a $31.7 \pm 5.3 \%$ reduction in the $\mathrm{SUV}_{\max }$ readings in the RAPA/BEV-treated mice compared to the control mice. Besides the drop in $\mathrm{SUV}_{\max }$ readings, it is also obvious 
a
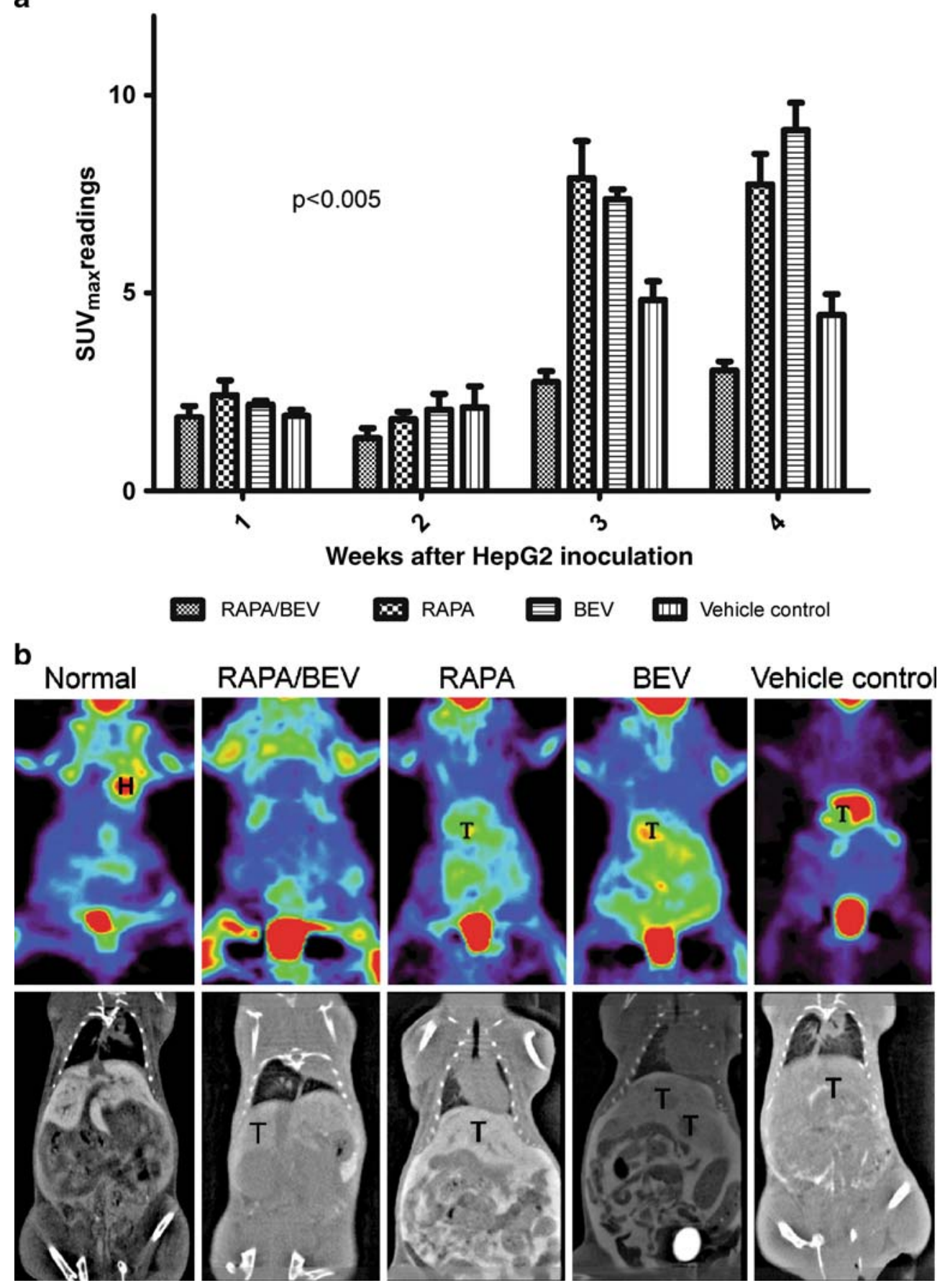

Fig. 2. a SUV $\mathrm{max}_{\max }$ readings of HCC over time. Mice were subjected to PET imaging weekly after tumor inoculation and their SUV $_{\text {max }}$ readings calculated. Error bars show SEM. b Representative PET and CT images of HCC mice with and without drug treatment. Top panels depict tumor metabolic activity by micro-PET imaging after a single dose administration of approximate $150 \mu \mathrm{Ci}$ of ${ }^{18} \mathrm{~F}$-FDG. Bottom panels show CT images after an intraperitoneal bolus dose of $20 \mathrm{ml} / \mathrm{kg}$ of Omnipaque 300 . Similar results were obtained for each of the 13 mice in each group.

from the PET images obtained that HCC mice undergoing combined RAPA/BEV chemotherapy exhibited less extensive spread of the tumor cells (Fig. 2b).

Similarly, CT imaging with Omnipaque reveals a welldefined liver in the normal mice. In the vehicle control animals, large tumor nodules were detected. In addition, large volumes of ascites were also observed. In contrast, the RAPA/BEV group had a smaller tumor volume on the CT image and almost no ascites were detected. Taken together, these possibly indicate that the drugs had a synergistic effect in slowing down the metabolic rate of HCC tumor cells and hence limiting its spread. 

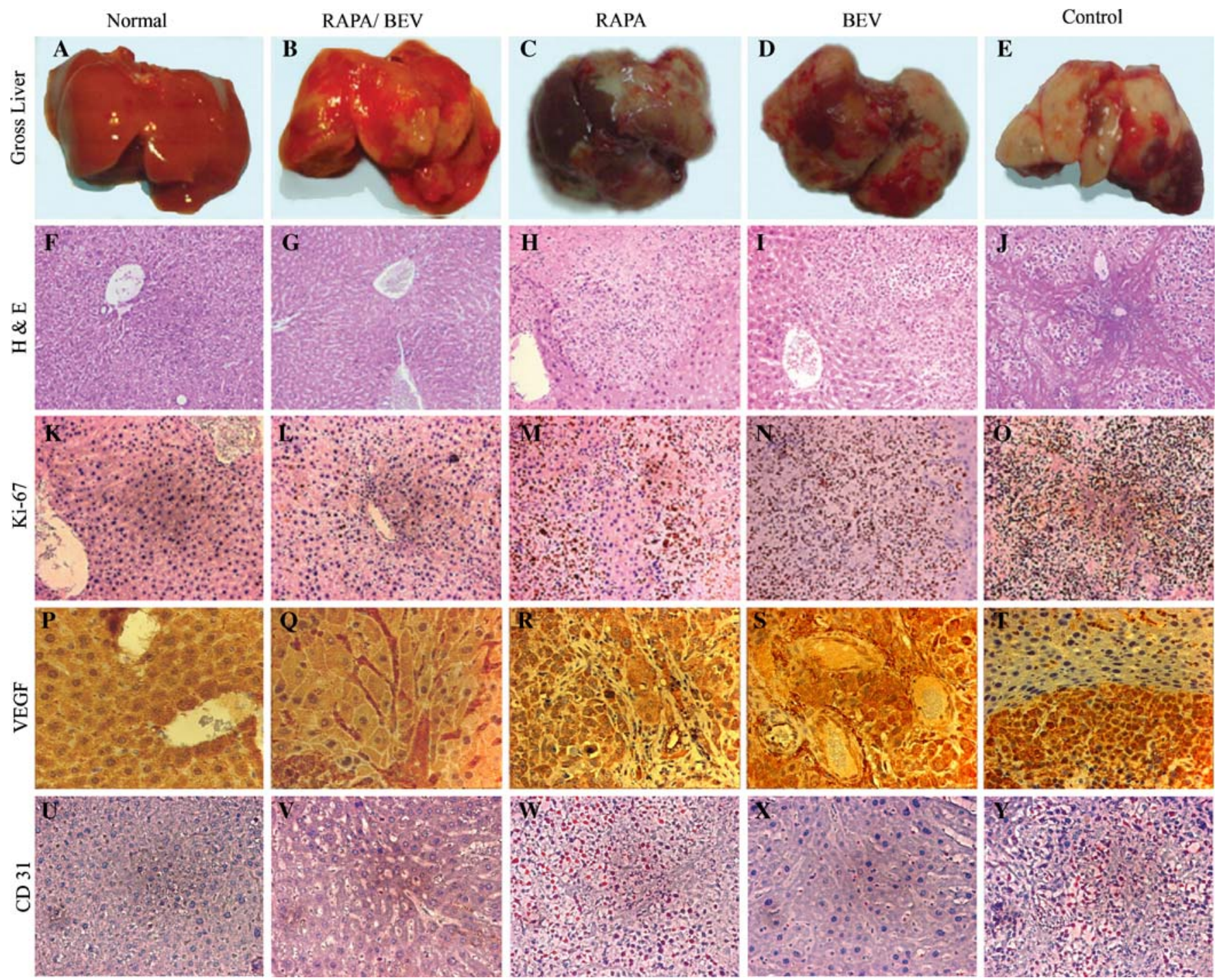

Fig. 3. Phenotypical effects and histological analysis of RAPA, BEV, and RAPA/BEV in HCC xenografts. Shown are representative livers $(\mathbf{a}-\mathbf{e})$ and histological sections $(\mathbf{f}-\mathbf{t})$ for HepG2 HCC xenografts. Xenografts were randomized into one of the four treatment groups and treated with vehicle (control), RAPA (1 mg/kg), BEV (5 mg/kg), or combined RAPA/BEV. All treatments were initiated 4 days after cell inoculation. a-e Effects on gross tumor morphology. $\mathbf{f}-\mathbf{j}$ Histological sections, stained with hematoxylin and eosin (H\&E) at $\times 20$ magnification. Tumors treated with single drug agent, BEV and RAPA, and vehicle control consisted mainly of dysplastic hepatocytes when compared to tumors treated with combined RAPA/BEV. Liver cirrhosis was also observed in the group treated with vehicle control. Immunohistochemical analysis of Ki-67 (k-0), and VEGF $(\mathbf{p}-\mathbf{t})$ and CD31 (u-y). Mice treated with RAPA/BEV showed the most dramatic decrease in Ki-67 (cell proliferation) when compared to vehicle control mice. VEGF protein was found to be expressed in both non-cancerous liver as well as HCC. VEGF expression is was stronger in well-differentiated HCC cells and dyplastic nodules than in normal hepatocytes. In poorly differentiated HCC, VEGF protein is expressed by the endothelial cells lining intratumoral vessels. CD31 was not detected in both the normal mice as well as mice treated with combined RAPA/BEV. In mice treated with single agent RAPA as well as those that received vehicle control, CD31 was expressed in the sinusoids of the HCC cells.

\section{The Use of RAPA/BEV Effectively Inhibits $H C C$ Xenografts as Depicted by Standard Histological Analysis}

To provide further support for the effectiveness of the use of combined RAPA/BEV treatment, we performed histological analysis on the dissected tumors (Fig. 3). Widespread necrosis was also predominantly found in the control mice but was minimal in the RAPA/BEV group. This also confirms our previous speculation that the lower $\mathrm{SUV}_{\text {max }}$ readings exhibited by the control group as compared to mice treated with only RAPA or BEV were due to large volume of necrotic liver.

Immunohistochemical analysis with Ki-67 revealed similar results as H\&E staining. Cell proliferation was most abundant in vehicle control mice followed by single drug 


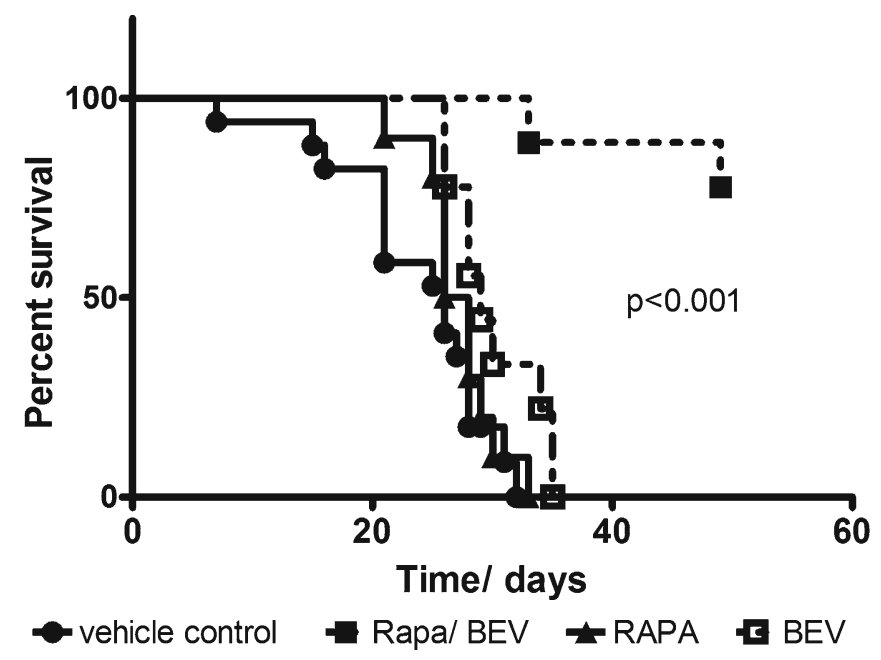

Fig. 4. Effects of RAPA/BEV on HCC xenograft mice. Each group comprised 13 animals. Kaplan-Meier analysis shows that mice with RAPA/BEV treatment had a significant higher survival rate than those that received either single-arm treatment or without treatment.

treatment mice. Control mice had a Ki-67 proliferation index of $69.8 \pm 4.6$ while mice treated with RAPA had an index of $46.4 \pm 10.1(p$ value $<0.05)$ and those treated with BEV had an index of $44.0 \pm 10.4$ ( $p$ value $<0.05)$. Mice that received the combined therapy had an almost twofold reduction in the index $(29.6 \pm 8.6, p$ value $<0.001)$.

On the other hand, VEGF expression was detected in both HCC cells as well as non-cancerous hepatocytes. VEGF was expressed faintly in the cytoplasm of hepatocytes of background liver. In the HCC cells, the expression of VEGF correlates negatively with the degree of cell differentiation. Mice that received the combined therapy had staining pattern that resembled closely to mice without HCC. In contrast, vehicle control mice showed strongly stained cells surrounded by layers of unstained HCC cells. In the BEV group, patches of strongly stained HCC cells were observed. Finally, mice treated with single agent RAPA had staining pattern intermediate of vehicle control mice and BEV-treated group.

In contrast to VEGF, CD31 was most abundantly expressed in the sinusoids of vehicle control mice. In mice that received either single agent therapy, CD31 was found to be expressed more in those treated with RAPA than those with BEV. This probably reflected sinusoidal capillarization, thus indicating the effectiveness of the respective drug therapy.

Hence, by combining the use of bioimaging techniques as well as histological analysis, we have shown that the combined RAPA/BEV chemotherapy is effective against HCC xenograft in the murine model.

\section{RAPA/BEV Inhibits Malignant Ascites and Prolongs Survival}

In this study, we developed an orthotopic intrahepatic model that strongly reflects clinical HCC. Within 3 weeks of tumor inoculation, control mice developed large volumes of ascites (3-4 ml). This was in sharp contrast with those that had received drug treatment. Unlike the control group, only one of the mice receiving RAPA/BEV treatment exhibited mild abdominal swelling at the end of week 5 . In the single drug treatment group, mice developed swollen abdomen only at week 4 and on dissection, an approximate volume of 1-2 ml of ascitic fluids could be extracted. Kaplan-Meier survival analysis (Fig. 4) provides further support for the efficacy of the drugs. RAPA/BEV-treated mice had a significantly prolonged overall survival. No significant effects on body weight, morbidity, or severe toxicity were observed in the RAPA/BEV treatment groups

\section{Discussion}

In this orthotopic xenograft model, mice treated with RAPA/ BEV had slower HCC growth and less ascites than untreated animals. Our results show that the combined use of rapamycin and bevacizumab prevents the progression of HCC and improves overall survival. The combined suppression of both VEGF activity by bevazicumab and VEGF expression via rapamycin probably induces both additive and synergistic inhibition on HCC growth and angiogenesis.

VEGF is known to play a significant role in the early stage of hepatocarcinogenesis although its exact mechanism remains unclear [1]. Its expression increases gradually from low-grade dysplastic nodules to high-grade dysplastic nodules to early HCC [24]. A possible mechanism by which VEGF is involved in liver carcinogenesis is VEGF synthesis by hepatocytes adjacent to the tumor nodule under the influence of tumor-released cytokines and growth factors [25]. Indeed, there have been numerous findings indicating VEGF over-expression in the cirrhotic liver of patients with a HCC, compared to cirrhotic livers without $\operatorname{HCC}[25,26]$. Hence, it is possible to target the proliferating endothelial cells instead of cancer cells. There are several advantages of antiangiogenic therapy over conventional cytotoxic chemotherapy. As microvascular endothelial cells are genetically stable cells that are less prone to mutation, drugs targeted at them are less likely than cytotoxic drugs to induce drug resistance. In addition, the problem of drug delivery to tumor cells can be easily overcome since endothelial cells are directly exposed to blood-borne agents. This is in sharp contrast with conventional anticancer therapy. Finally, due to the specificity of the antiangiogenic therapy towards immature characteristics of tumor vasculature, which differs from normal quiescent vasculature, low toxicity has been demonstrated in pre-clinical studies [27].

There are now increasing evidence in pre-clinical studies that antiangiogenic therapy using different approaches can inhibit the growth of HCC in synergistic manners. Recent clinical trials have shown the efficacy of bevacizumab in combination with chemotherapy in treating various types of human cancers [28-30]. In a recent report by Semela and coworkers, they have concluded using an orthotopic, 
syngenic model that rats treated with rapamycin exhibited blockage of tumor angiogenesis and impaired HCC growth [31]. In our study, we combined the use of both bevacizumab and rapamycin. We speculate that besides suppressing VEGF expression from HCC tumor cells, rapamycin might possibly inhibit proliferation of endothelial cells and blocked angiogenesis. As such, the use of a combined RAPA/BEV might provide a potent antiangiogenic therapy for retardation of HCC progression. Many studies have suggested that HCC originates as a well-differentiated tumor and becomes progressively less differentiated with enlargement [32]. Our immunohistochemistry staining of VEGF showed that only the vehicle control mice eventually developed advanced HCC characterized by poorly differentiated HCC tissues surrounded by well-differentiated HCC tissues. Although mice treated with either single agent did not exhibit advanced HCC, they nonetheless displayed "patchy" strong expression of VEGF, especially in mice treated with RAPA only. All these results further strengthened our hypothesis that combined RAPA/BEV is effective in preventing HCC progression.

Our paper is also novel in that it compares the diagnostic values of micro-PET/CT and conventional histological analysis. Although histological analysis is extremely useful and accurate at diagnosing or even staging diseases such as $\mathrm{HCC}$, its procedures are usually long and tedious and require expertise to interpret the results. This is true in clinical practice as well. Despite being the most definite assessment of HCC grading, histological examination using percutaneous needle biopsy is invasive and the specimen retrieved does not always represent the entire lesion due to sampling errors [33]. Moreover, the prognosis and survival of patients with HCC is heavily dependent on the disease stage at the time of diagnosis. Currently, alpha-fetoprotein (AFP) is the only serological marker that is available for the detection of HCC clinically. Although minimally invasive, it is not a sufficiently reliable marker because of its poor sensitivity [34]. Moreover, its level varies between ethnic groups and is also not recommended for diagnosis of $\mathrm{HCC}$ in non-cirrhotic patients [35, 36]. In a study by Giannelli et al. [34], only a small percentage of patients showed AFP values that are reflective of HCC. Several other reports have also indicated that the AFP sensitivity range from $47 \%$ to $68 \%$ [37].

On the other hand, the use of bioimaging modalities overcomes all these obstacles. Compared with conventional single bioimaging modality such as CT, MRI, or ultrasonography, dual PET/CT with ${ }^{18} \mathrm{~F}-\mathrm{FDG}$ has advantages in the evaluation of hepatic malignancies, including diagnosing, staging tumors, evaluating biologic characters, and monitoring tumor progression. By offering a combined functional and anatomical analysis, PET/CT has been increasingly used in clinical practice since it is more sensitive and specific than either PET or CT alone [38]. Salem and coworkers have recently reported the correlation between ${ }^{18} \mathrm{~F}$-FDG uptake in woodchuck HCCs and the tumor differentiation level [31]. Similarly, our results derived from micro-PET/CT also corresponded with that of standard histology analysis. Such findings have great impact on future experimental design. With the use of bioimaging modalities such as micro-PET and micro-CT, histological analysis at every stage is no longer critical, allowing serial follow-up and assessment of each animal into the last stages of the disease.

The objectives of this study were to evaluate the efficacy of the combined RAPA/BEV antiangiogenic therapy as well as to assess the performance of ${ }^{18} \mathrm{~F}$-FDG-PET in an orthotopic xenograft HCC model. Our results clearly illustrate that pharmacological inhibition of the mTOR with rapamycin and specific targeting of VEGF with bevacizumab may provide an effective novel treatment for HCC. In addition, the use of micro-PET/CT may prove to be an excellent research tool to monitor progression of orthotopic cancer models in testing therapy efficacies.

Open Access. This article is distributed under the terms of the Creative Commons Attribution Noncommercial License which permits any noncommercial use, distribution, and reproduction in any medium, provided the original author(s) and source are credited.

\section{References}

1. Pang R, Poon RT (2006) Angiogenesis and antiangiogenic therapy in hepatocellular carcinoma. Cancer Lett 242:151-167

2. Parkin DM, Bray F, Ferlay J, Pisani P (2001) Estimating the world cancer burden: Globocan 2000. Int J Cancer 94:153-156

3. Llovet JM (2004) Treatment of hepatocellular carcinoma. Curr Treat Options Gastroenterol 7:431-441

4. Poon RT, Fan ST (2004) Resection prior to liver transplantation for hepatocellular carcinoma: a strategy of optimizing the role of resection and transplantation in cirrhotic patients with preserved liver function. Liver Transpl 10:813-815

5. Nowak AK, Chow PK, Findlay M (2004) Systemic therapy for advanced hepatocellular carcinoma: a review. Eur J Cancer 40:14741484

6. Rao RD, Buckner JC, Sarkaria JN (2004) Mammalian target of rapamycin (mTOR) inhibitors as anti-cancer agents. Curr Cancer Drug Targets 4:621-635

7. Fingar DC, Blenis J (2004) Target of rapamycin (TOR): an integrator of nutrient and growth factor signals and coordinator of cell growth and cell cycle progression. Oncogene 23:3151-3171

8. Fingar DC, Richardson CJ, Tee AR, Cheatham L, Tsou C, Blenis J (2004) mTOR controls cell cycle progression through its cell growth effectors S6K1 and 4E-BP1/eukaryotic translation initiation factor 4E. Mol Cell Biol 24:200-216

9. Guba M, von Breitenbuch P, Steinbauer M, Koehl G, Flegel S, Hornung $M$ et al (2002) Rapamycin inhibits primary and metastatic tumor growth by antiangiogenesis: involvement of vascular endothelial growth factor. Nat Med 8:128-135

10. McCarthy M (2003) Antiangiogenesis drug promising for metastatic colorectal cancer. Lancet 361:1959

11. Yang JC, Haworth L, Sherry RM, Hwu P, Schwartzentruber DJ, Topalian SL et al (2003) A randomized trial of bevacizumab, an antivascular endothelial growth factor antibody, for metastatic renal cancer. N Engl J Med 349:427-434

12. Kerbel R, Folkman J (2002) Clinical translation of angiogenesis inhibitors. Nat Rev Cancer 2:727-739

13. Sausville EA, Burger AM (2006) Contributions of human tumor xenografts to anticancer drug development. Cancer Res 66:33513354, discussion 3354

14. Teicher BA (2006) Tumor models for efficacy determination. Mol Cancer Ther 5:2435-2443

15. Johnson JI, Decker S, Zaharevitz D, Rubinstein LV, Venditti JM, Schepartz S et al (2001) Relationships between drug activity in NCI preclinical in vitro and in vivo models and early clinical trials. Br J Cancer 84:1424-1431 
16. Chang CJ, Tai KF, Roffler S, Hwang LH (2004) The immunization site of cytokine-secreting tumor cell vaccines influences the trafficking of tumor-specific $\mathrm{T}$ lymphocytes and antitumor efficacy against regional tumors. J Immunol 173:6025-6032

17. Bibby MC (2004) Orthotopic models of cancer for preclinical drug evaluation: advantages and disadvantages. Eur J Cancer 40:852-857

18. Wahl RL, Hutchins GD, Buchsbaum DJ, Liebert M, Grossman HB, Fisher S (1991) 18F-2-deoxy-2-fluoro-D-glucose uptake into human tumor xenografts. Feasibility studies for cancer imaging with positronemission tomography. Cancer 67:1544-1550

19. Strauss LG, Conti PS (1991) The applications of PET in clinical oncology. J Nucl Med 32:623-648, discussion 649-650

20. Deroose CM, De A, Loening AM, Chow PL, Ray P, Chatziioannou AF et al (2007) Multimodality imaging of tumor xenografts and metastases in mice with combined small-animal PET, small-animal CT, and bioluminescence imaging. J Nucl Med 48:295-303

21. Jeng LB, Changlai SP, Shen YY, Lin CC, Tsai CH, Kao CH (2003) Limited value of $18 \mathrm{~F}$-2-deoxyglucose positron emission tomography to detect hepatocellular carcinoma in hepatitis B virus carriers. Hepatogastroenterology 50:2154-2156

22. Khan MA, Combs CS, Brunt EM, Lowe VJ, Wolverson MK, Solomon $\mathrm{H}$ et al (2000) Positron emission tomography scanning in the evaluation of hepatocellular carcinoma. J Hepatol 32:792-797

23. Ho CL, Chen S, Yeung DW, Cheng TK (2007) Dual-tracer PET/CT imaging in evaluation of metastatic hepatocellular carcinoma. J Nucl Med 48:902-909

24. Atkin G, Barber PR, Vojnovic B, Daley FM, Glynne-Jones R, Wilson GD (2005) Correlation of spectral imaging and visual grading for the quantification of thymidylate synthase protein expression in rectal cancer. Hum Pathol 36:1302-1308

25. Park YN, Kim YB, Yang KM, Park C (2000) Increased expression of vascular endothelial growth factor and angiogenesis in the early stage of multistep hepatocarcinogenesis. Arch Pathol Lab Med 124:1061-1065

26. An FQ, Matsuda M, Fujii H, Matsumoto Y (2000) Expression of vascular endothelial growth factor in surgical specimens of hepatocellular carcinoma. J Cancer Res Clin Oncol 126:153-160

27. Eskens FA, Verweij J (2006) The clinical toxicity profile of vascular endothelial growth factor (VEGF) and vascular endothelial growth factor receptor (VEGFR) targeting angiogenesis inhibitors; a review. Eur J Cancer 42:3127-3139
28. Hicklin DJ, Ellis LM (2005) Role of the vascular endothelial growth factor pathway in tumor growth and angiogenesis. J Clin Oncol 23:1011-1027

29. Johnson DH, Fehrenbacher L, Novotny WF, Herbst RS, Nemunaitis JJ, Jablons DM et al (2004) Randomized phase II trial comparing bevacizumab plus carboplatin and paclitaxel with carboplatin and paclitaxel alone in previously untreated locally advanced or metastatic non-small-cell lung cancer. J Clin Oncol 22:2184-2191

30. Hurwitz HI, Fehrenbacher L, Hainsworth JD, Heim W, Berlin J, Holmgren E et al (2005) Bevacizumab in combination with fluorouracil and leucovorin: an active regimen for first-line metastatic colorectal cancer. J Clin Oncol 23:3502-3508

31. Semela D, Piguet AC, Kolev M, Schmitter K, Hlushchuk R, Djonov V et al (2007) Vascular remodeling and antitumoral effects of mTOR inhibition in a rat model of hepatocellular carcinoma. J Hepatol 46:840 848

32. Torimura T, Sata M, Ueno T, Kin M, Tsuji R, Suzaku K et al (1998) Increased expression of vascular endothelial growth factor is associated with tumor progression in hepatocellular carcinoma. Hum Pathol 29:986-991

33. Sun L, Wu H, Pan WM, Guan YS (2007) Positron emission tomography/computed tomography with (18)F-fluorodeoxyglucose identifies tumor growth or thrombosis in the portal vein with hepatocellular carcinoma. World J Gastroenterol 13:4529-4532

34. Giannelli G, Fransvea E, Trerotoli P, Beaugrand M, Marinosci F, Lupo $\mathrm{L}$ et al (2007) Clinical validation of combined serological biomarkers for improved hepatocellular carcinoma diagnosis in 961 patients. Clin Chim Acta 383:147-152

35. Nguyen MH, Garcia RT, Simpson PW, Wright TL, Keeffe EB (2002) Racial differences in effectiveness of alpha-fetoprotein for diagnosis of hepatocellular carcinoma in hepatitis $\mathrm{C}$ virus cirrhosis. Hepatology 36:410-417

36. Cedrone A, Covino M, Caturelli E, Pompili M, Lorenzelli G, Villani MR et al (2000) Utility of alpha-fetoprotein (AFP) in the screening of patients with virus-related chronic liver disease: does different viral etiology influence AFP levels in HCC? A study in 350 western patients. Hepatogastroenterology 47:1654-1658

37. Marrero JA, Lok AS (2004) Newer markers for hepatocellular carcinoma. Gastroenterology 127:S113-S119

38. von Schulthess GK, Steinert HC, Hany TF (2006) Integrated PET/CT: current applications and future directions. Radiology 238:405-422 